\title{
$R P 1$ and autosomal dominant rod-cone dystrophy: novel mutations, a review of published variants, and genotype-phenotype correlation
}

Isabelle Audo ${ }^{1,2,3,4,5}$, Saddek Mohand-Saïd ${ }^{1,2,3,4}$, Claire-Marie Dhaenens ${ }^{6}$, Aurore

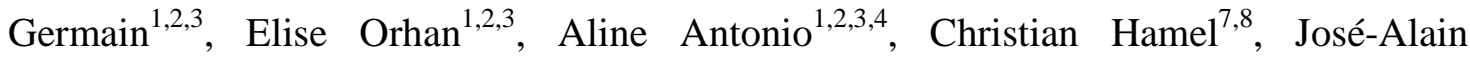
Sahel $^{1,2,3,4,5,9}$, Shomi S. Bhattacharya ${ }^{1,2,3,5,10}$ and Christina Zeitz ${ }^{1,2,3}$.

${ }^{1}$ INSERM, UMRS968, Paris, F-75012, France; ${ }^{2}$ UPMC Univ Paris 06, UMR_S 968, Institut de la Vision, Paris, F-75012, France; ${ }^{3}$ CNRS, UMR_7210, Paris, F-75012, France; ${ }^{4}$ Centre Hospitalier National d'Ophtalmologie des Quinze-Vingts, INSERMDHOS CIC 503, Paris, F-75012, France; ${ }^{5}$ UCL-Institute of Ophthalmology, Bath Street, London, UK ; ${ }^{6}$ Centre Hospitalier Régional Universitaire de Lille, UF Génopathies, Laboratoire de Biochimie et Biologie Moléculaire - Université Lille Nord de France, Lille, France ; ${ }^{7}$ Centre de Référence Maladies Sensorielles Génétiques, Hôpital Guy de Chauliac, Montpelier, France; ${ }^{8}$ U583, INSERM, Institute for Neurosciences de Montpellier, Montpellier, France; ${ }^{9}$ Fondation Ophtalmologique Adolphe de Rothschild, Paris, France; ${ }^{10}$ Department of Celular Therapy and Regenerative Medicine, Andalusian Molecular Biology and Regenerative Medicine Centre (CABIMER), Seville, Spain.

Running title: $R P 1$ mutations in French adRP patients

Key words: adRP, $R P 1$ mutations, prevalence study

Corresponding author: Isabelle Audo and Christina Zeitz

Institut de la Vision, Department of Genetics

17, Rue Moreau

75012 Paris

France

Email address: isabelle.audo@inserm.fr, christina.zeitz@inserm.fr 
Financial support:

The project was financially supported by the Department of Paris, Foundation Fighting Blindness (I.A. FFB Grant N: CD-CL-0808-0466-CHNO and the CIC503 recognized as an FFB center, FFB Grant No: C-CMM-0907-0428-INSERM04), ANR (S.S.B). NIHR Biomedical Research Centre for Ophthalmology and The Special Trustees of Moorfields Eye Hospital London, Foundation Voir et Entendre (C.Z) and French Ministry of Health (C.H. PHRC \# 2008-A01238-47). 


\begin{abstract}
Rod-cone dystrophies (RP) are a clinically and genetically heterogeneous group of inherited retinal disorders characterized by photoreceptor degeneration. $R P 1$ is a major gene underlying autosomal dominant (ad) RP though prevalence numbers vary depending on the origin of the cases from $0 \%-10 \%$ cases. Some mutations in $R P 1$ also lead to autosomal recessive RP. Herein we review all previously reported and several novel RPI mutations in relation to the associated phenotype in patients from a French adRP cohort. Prevalence studies from this cohort show that $5.3 \%$ of the cases have $R P 1$ mutations. This is in accordance with other studies reported from UK and USA. The majority of mutations represent truncating mutations which are located in a hot spot region. Similarly, we identified in total four novel deletions and nonsense mutations, of which two may represent recurrent mutations in a French population. In addition a novel missense mutation of uncertain pathogenicity was identified. With our findings, to date 43 RP1 mutations are known to cause adRP. Variable penetrance of the disease was observed in our and other cohorts. Most patients with $R P 1$ mutations show classical signs of RP with relatively preserved central vision and visual field.
\end{abstract}




\section{Background}

Rod-cone dystrophies, also known as retinitis pigmentosa (RP), are a clinically and genetically heterogeneous group of progressive inherited retinal disorders, which often starts with night blindness and leads to visual field constriction and secondary macular involvement, which can eventually result in loss of central vision and complete blindness (Hartong et al. 2006). RP occurs in 1 of 4000 births and affects more than 1 million individuals worldwide. The mode of inheritance can be $\mathrm{x}$-linked (xl), autosomal dominant (ad) or autosomal recessive (ar). In addition, many patients represent isolated cases, due to the absence of family history of RP. To date, mutations in 23 different genes are associated with autosomal dominant RP (adRP) (http://www.sph.uth.tmc.edu/Retnet/) and the majority of prevalence studies reveals rhodopsin (RHO: MIM\#180380) being the most frequently mutated gene in adRP (Sullivan et al. 2006; Audo et al. 2010b). In addition, PRPF31(MIM\#606419), PRPH2 (MIM\#179605) and RP1 (MIM\#603937) were proposed to represent major genes underlying this form of RP (Sullivan et al. 2006; Audo et al. 2010a). In some rare cases $R P 1$ mutations were also found in patients with arRP and unilateral RP (Khaliq et al. 2005; Riazuddin et al. 2005; Singh et al. 2009; Chen et al. 2010; Mukhopadhyay et al. 2011). $R P 1$ is located on chromosome 8q12.1, encompasses 4 exons of which 3 are coding. This work aims to review known mutations and to document novel mutations and genotype-phenotype correlations on a French cohort consisting of 114 cases. 


\section{Mutations in the RP1 gene}

So far 39 disease causing mutations in $R P 1$ have been identified of which most were found in the last exon, leading to a premature stop codon and are predicted to form a truncated protein (Table 1). The p.Arg677X has been described as the most commonly reported mutation (Bowne et al. 1999; Guillonneau et al. 1999; Pierce et al. 1999; Sullivan et al. 1999; Jacobson et al. 2000; Payne et al. 2000; Baum et al. 2001; Berson et al. 2001; Sohocki et al. 2001; Xiaoli et al. 2002; Schwartz et al. 2003; Ziviello et al. 2005; Chiang et al. 2006; Gamundi et al. 2006; Roberts et al. 2006; Sullivan et al. 2006).. Detailed phenotype-genotype correlations of patients with this mutation revealed incomplete penetrance and high variability of the disease expression in adRP suggesting modifiers to be involved (Jacobson et al. 2000). Similarly, incomplete penetrance was also noted for other mutations (Table 1). Mutation analysis in our cohort (85 of 114 adRP index patients screened for $R P 1$ ) added two different deletions and two different nonsense mutations identified in 6 families to the previously described mutations, however none of the patients revealed the recurrent p.Arg677X mutation (Table 1, Supp. Table S1).With our findings, to date $43 R P 1$ mutations are known to cause adRP. The prevalence of $R P 1$ mutations with $5,3 \%$ in our cohort is similar to studies from UK and USA, although the prevalence of $R P 1$ mutations varied from $0 \%-10 \%$ from different adRP cohorts (Bowne et al. 1999; Pierce et al. 1999; Payne et al. 2000; Baum et al. 2001; Berson et al. 2001; Sohocki et al. 2001; Kawamura et al. 2004; Ziviello et al. 2005; Gamundi et al. 2006; Roberts et al. 2006; Sullivan et al. 2006; Zhang et al. 2010) 
Different pathogenic mechanisms of $R P 1$ truncating mutations have been proposed due to in vitro and in vivo studies leading to ar or adRP. Chen and co-workers proposed four classes of $R P 1$ mutations depending on their site and underlying pathogenicity (Chen et al. 2010). Class 1 mutations are rare, thought to be nonsense-mediated decay (NMD)sensitive and locate in exons 2-3 (amino acids 1-236). Haploinsufficiency is not believed to be the disease causing mechanism since heterozygous carriers of those mutations are not affected. Thus it is more likely that the mutation creates a null allele due to NMD. The RP1 protein made by the unaffected copy of the allele is completely functional and only if both alleles are affected, the patient will suffer from RP. Examples are p.Ser2ArgfsX16 (Chen et al. 2010) and p.Pro229GlnfsX35 (Pierce et al. 2010) (Table 1). Class II mutations occur frequently and are reported to be NMD-insensitive truncations, which are located approximately between amino acids 500 and 1053 in exon 4 . The mutant proteins are expected to impose distinct dominant negative effect on photoreceptors, resulting in cell death and adRP (Chen et al. 2010) (Table 1). Similarly, the deletions and nonsense mutations identified in the French adRP cohort studied herein could also be classified as class II mutations. Two of them (p.Lys657AsnfsX7 and p.Ile725TyrfsX13) may represent re-current mutations in French adRP patients due to the appearance in two independent families (Table 1, Supp. Figure S1, Supp. Table S1). To our knowledge only one recessive truncating mutation lies in this region and leads to arRP (p.Asn949LysfsX32) (Table 1). Class III truncating mutations are thought to affect amino acids 264 to 499 and 1054 to 1751 , which lead to loss-of-function in patients with arRP. Examples are p.Pro1648SerX13 (Chen et al. 2010) or p.Asn1751IlefsX4 
(Riazuddin et al. 2005) (Table 1). Class IV truncating mutations are NMD-insensitive and thought to be located after amino acid 1816 . The resulting truncated proteins are expected to be functional and thus not disease causing (Chen et al. 2010). A few missense mutations reported lead to ar or adRP (Table 1) (Khaliq et al. 2005; Chiang et al. 2006; Zhang et al. 2010). However for most of the missense mutations the pathogenic character remains to be elucidated or was considered to be benign (Supp. Tables S2 and S3). Similarly, we identified a missense mutation (c.4193C >G, p.Ser1398Cys, Supp. Figure $\mathrm{S} 1, \mathrm{~F} 745$,) of which co-segregation analysis can not clearly predict if this variant lead to adRP (Supp. Table S4). Furthermore other variants in $R P 1$ have been identified, which are presumably due to predictions or co-segregation analysis not pathogenic (Supp. Table S5). All mutations identified by our extensive literature review and described herein, will be submitted to the existing Locus Specific Databses (LSDB) on RP1, which before the submission of our review cites only 4 pathogenic mutations.

\section{Genotype-Phenotype correlation}

Variable penetrance of the disease was observed in our and other cohorts. Most patients with $R P 1$ mutations show classical signs of RP with relatively preserved central vision and visual field. The detailed description about the phenotypic characteristics of the 7 index patients ( 3 females and 4 males) carrying different $R P 1$ mutations of the French adRP cohort can be used as key insights about $R P 1$ associated genotype-phenotype relationships (Table 2 and 3 with an illustration of retinal changes presented in figure 1). In brief, mean age of patients of the patients herein is 54.4. Except for one patient, 
CIC01234 (carries the missense mutation of uncertain pathogenicity) examined at an asymptomatic young age during a family investigation, the majority of subjects were diagnosed in their twenties with an average age at time of diagnosis of about 23 years. This is older than reported in adRP associated with $R H O$ and PRPF31 mutations (Audo et al. 2010a; Audo et al. 2010b) and in accordance with previous reports for patients with RP1 mutations (Berson et al. 2001). Family history for two families (F556 and F745) suggested incomplete penetrance and three patients reported variable age of onset in the family with diagnosis made as late as 55 years. This is in keeping with variable penetrance and expressivity associated with $R P 1$ mutations (Jacobson et al. 2000; Berson et al. 2001). None of the families in this study mentioned unilateral involvement so far (Mukhopadhyay et al. 2011). Symptoms at presentation were dominated by night vision disturbances. Visual acuity ranges from $20 / 25$ to 20/800. All patients had some lens opacities or had undergone cataract surgery. In most cases, fundus changes were typical of RP with narrowed retinal vessels, pale discs and pigmentary changes in the periphery. All but one had some degree of atrophy with loss of autofluorescence in the perifoveal region and one patient also had cystoid macular oedema in one eye. Color vision abnormalities were correlated with worse visual acuities. All patients had severe constricted visual fields and generalized retinal dysfunction with four out of seven with some preservation of central macular function. Therefore, although phenotypic variability was suggested in certain families in this study, the index patients had a fairly homogeneous degree of severity within their retinal involvement. 


\section{Biological Relevance}

RP1 consists of 2156 amino acids with a molecular weight of $240 \mathrm{kD}$, which is expressed specifically in photoreceptors in the retina (Bowne et al. 1999; Guillonneau et al. 1999; Pierce et al. 1999). The N-terminal 28-228 amino acids of RP1 contains a tandem repeat domain (Kim et al. 2003), which is known to interact with microtubules and was initially detected in the protein doublecortin (DCX). DCX is involved in directing neuronal migration during development of the central nervous system and mutations in this gene in patients lead to x-linked lissencephaly and double cortex syndrome (des Portes et al. 1998; Gleeson et al. 1998; Francis et al. 1999). Immunofluorescence analyses detected the human and mouse RP1 protein in the connecting cilium and microtubule-based axoneme of rod and cone photoreceptors. In addition, the amino acid sequences between codons p.486 and p.635 shares homology with the Drosophila melangonaster protein BIF, which is essential for normal photoreceptor morphogenesis (Pierce et al. 1999). Due to the specific localization of RP1 and the existence of the typical DCX and BIF domain, it was suggested that RP1 might participate in transport of proteins between the inner and outer segments of photoreceptors or in maintenance of the ciliary structure (Liu et al. 2002). Different mouse lines lacking Rp1 have been created to better understand the function and pathogenic mechanism of RP1 protein and mutations: One deletes the conserved N-terminal Dcx domain (Gao et al. 2002) and produces an abnormal Rp1 product that is lacking exon 2 and 3, while the other mimics the frequently identified mutation in RP patients: p.Arg677X, which contains the N-terminal Dcx domain and produces the shorter Rp1 protein (Liu et al. 2003). Both homozygous models experience 
rapid retinal degeneration and show outer segment dysplasia and misalignment, suggesting a general role of RP1 in outer segment organization. Initially, only heterozygous mice lacking the Dcx domain and expressing about $50 \%$ of normal Rp1 protein revealed some retinal dysfunction despite normal-appearing retinal morphology (Gao et al. 2002), while heterozygous mice mimicking the human mutation p.Arg677X did not show any phenotype (Liu et al. 2003). However, recently it was shown that the severity of the retinal degeneration is dependent on the genetic background and only heterozygous mice for the A.129S(B6) background indeed developed RP suggesting that specific modifiers present in one or the other mouse strain are responsible for the severity of the phenotype in some heterozygous mouse lines (Liu et al. 2009). Furthermore the photoreceptor degeneration of a knock-in mouse line carrying the p.Gln662X mutation, analogous to the human p.Arg677X mutation, could be delayed or prevented by adding wild-type Rp1 protein (Pierce et al. 2010). Another mouse model, which was chemically induced $(\mathrm{N}-$-ethyl-N-nitrosourea $=$ ENU) carries a p.Arg533X mutation and again only homozygous mice were affected by photoreceptor degeneration (Won et al. 2011). Since the retinal phenotype in knock-in mice carrying mutations in this region could be delayed or prevented by adding the wild-type gene, the underlying pathogenic mechanism was suggested to be dominant-negative and not gain-of-function.

\section{Clinical and Diagnostic Relevance}

In the study presented here we review all reported disease causing $R P 1$ mutations including 4 novel truncating mutations and 1 novel nucleotide change of uncertain 
pathogenicity in 7 cases with a homogenous RP phenotype (Table 1, 2 and 3, Supp. Tables S1 and S2). Our study confirms that for adRP European cohorts, $R P 1$ represents a major gene defect. We propose that most of the $R P 1$ mutations leading to adRP represent truncation mutations and are located in a hot spot region ( nucleotides c.1490-c.3216). Therefore, to perform a time and cost-efficient mutations screening for adRP patients this region could be preferentially targeted. These truncating mutations can be classified as Class II mutations, which lead to a dominant negative effect of $R P 1$ resulting in photoreceptor cell degeneration. In addition, incomplete penetrance was observed for some of the mutations in this class (Dietrich et al. 2002; Roberts et al. 2006) (Table 1). The exact mechanism of this incomplete penetrance is not known. Another form of adRP associated with mutation in splicing factors is also associated with incomplete penetrance (Evans et al. 1995; Al-Maghtheh et al. 1996; McGee et al. 1997; Vaclavik et al. 2010). In this case, haploinsufficiency is the admitted disease mechanism and an allelic imbalance with overexpression of the wild-type allele, compensating for the non-functional allele in asymptomatic carriers has been advocated (Vithana et al. 2003; Rivolta et al. 2006). In case of incomplete penetrance associated with $R P 1$ mutation, such mechanisms with the influence of the trans allele has been suggested in asymptomatic female carriers by Daiger and colleagues but warrants further study (Daiger et al. 2006). Influence of mouse strains of $R p l$ mutation upon severity in retinal degeneration advocate for modifying factors (Liu et al. 2009). A better understanding of the mechanisms underlying phenotypic variability should facilitate developing novel therapeutic approaches. 
Clinically and genetically well-characterized patients such as those included here are critical in this regard.

\section{ACKNOWLEDGEMENT:}

The authors are grateful to patients and family members described in this study, to Thierry Léveillard, Dominique Santiard-Baron, Christine Chaumeil and clinical staff for their help in clinical data and DNA collection. The project was financially supported by the Department of Paris, Foundation Fighting Blindness (I.A. FFB Grant N ${ }^{\circ}$ : CD-CL0808-0466-CHNO and the CIC503 recognized as an FFB center, FFB Grant №: CCMM-0907-0428-INSERM04), ANR (S.S.B). NIHR Biomedical Research Centre for Ophthalmology and The Special Trustees of Moorfields Eye Hospital London, Foundation Voir et Entendre (C.Z) and French Ministry of Health (C.H. PHRC \# 2008A01238-47).

\section{REFERENCES:}

Al-Maghtheh M, Vithana E, Tarttelin E, Jay M, Evans K, Moore T, Bhattacharya S, Inglehearn CF. 1996. Evidence for a major retinitis pigmentosa locus on 19q13.4 (RP11) and association with a unique bimodal expressivity phenotype. Am J Hum Genet 59(4):864-71.

Audo I, Bujakowska K, Mohand-Said S, Lancelot ME, Moskova-Doumanova V, Waseem NH, Antonio A, Sahel JA, Bhattacharya SS, Zeitz C. 2010a. Prevalence and novelty of PRPF31 mutations in French autosomal dominant rod-cone dystrophy patients and a review of published reports. BMC Med Genet 11:145.

Audo I, Manes G, Mohand-Said S, Friedrich A, Lancelot ME, Antonio A, MoskovaDoumanova V, Poch O, Zanlonghi X, Hamel CP, Sahel JA, Bhattacharya SS, Zeitz C. 2010b. Spectrum of rhodopsin mutations in French autosomal dominant rod-cone dystrophy patients. Invest Ophthalmol Vis Sci published online 17.02.2010.

Baum L, Chan WM, Yeung KY, Lam DS, Kwok AK, Pang CP. 2001. RP1 in Chinese: Eight novel variants and evidence that truncation of the extreme C-terminal does not cause retinitis pigmentosa. Hum Mutat 17(5):436. 
Berson EL, Grimsby JL, Adams SM, McGee TL, Sweklo E, Pierce EA, Sandberg MA, Dryja TP. 2001. Clinical features and mutations in patients with dominant retinitis pigmentosa-1 (RP1). Invest Ophthalmol Vis Sci 42(10):2217-24.

Bowne SJ, Daiger SP, Hims MM, Sohocki MM, Malone KA, McKie AB, Heckenlively JR, Birch DG, Inglehearn CF, Bhattacharya SS, Bird A, Sullivan LS. 1999. Mutations in the RP1 gene causing autosomal dominant retinitis pigmentosa. Hum Mol Genet 8(11):2121-8.

Chen LJ, Lai TY, Tam PO, Chiang SW, Zhang X, Lam S, Lai RY, Lam DS, Pang CP. 2010. Compound heterozygosity of two novel truncation mutations in RP1 causing autosomal recessive retinitis pigmentosa. Invest Ophthalmol Vis Sci 51(4):2236-42.

Chiang SW, Wang DY, Chan WM, Tam PO, Chong KK, Lam DS, Pang CP. 2006. A novel missense RP1 mutation in retinitis pigmentosa. Eye (Lond) 20(5):602-5.

Daiger SP, Shankar SP, Schindler AB, Sullivan LS, Bowne SJ, King TM, Daw EW, Stone EM, Heckenlively JR. 2006. Genetic factors modifying clinical expression of autosomal dominant RP. Adv Exp Med Biol 572:3-8.

des Portes V, Pinard JM, Billuart P, Vinet MC, Koulakoff A, Carrie A, Gelot A, Dupuis E, Motte J, Berwald-Netter Y, Catala M, Kahn A, Beldjord C, Chelly J. 1998. A novel CNS gene required for neuronal migration and involved in $\mathrm{X}$-linked subcortical laminar heterotopia and lissencephaly syndrome. Cell 92(1):51-61.

Dietrich K, Jacobi FK, Tippmann S, Schmid R, Zrenner E, Wissinger B, Apfelstedt-Sylla E. 2002. A novel mutation of the RP1 gene (Lys778ter) associated with autosomal dominant retinitis pigmentosa. Br J Ophthalmol 86(3):328-32.

Evans K, al-Maghtheh M, Fitzke FW, Moore AT, Jay M, Inglehearn CF, Arden GB, Bird AC. 1995. Bimodal expressivity in dominant retinitis pigmentosa genetically linked to chromosome 19q. Br J Ophthalmol 79(9):841-6.

Francis F, Koulakoff A, Boucher D, Chafey P, Schaar B, Vinet MC, Friocourt G, McDonnell N, Reiner O, Kahn A, McConnell SK, Berwald-Netter Y, Denoulet P, Chelly J. 1999. Doublecortin is a developmentally regulated, microtubuleassociated protein expressed in migrating and differentiating neurons. Neuron 23(2):247-56.

Gamundi MJ, Hernan I, Martinez-Gimeno M, Maseras M, Garcia-Sandoval B, Ayuso C, Antinolo G, Baiget M, Carballo M. 2006. Three novel and the common Arg677Ter RP1 protein truncating mutations causing autosomal dominant retinitis pigmentosa in a Spanish population. BMC Med Genet 7:35.

Gao J, Cheon K, Nusinowitz S, Liu Q, Bei D, Atkins K, Azimi A, Daiger SP, Farber DB, Heckenlively JR, Pierce EA, Sullivan LS, Zuo J. 2002. Progressive photoreceptor degeneration, outer segment dysplasia, and rhodopsin mislocalization in mice with targeted disruption of the retinitis pigmentosa-1 (Rp1) gene. Proc Natl Acad Sci U S A 99(8):5698-703.

Gleeson JG, Allen KM, Fox JW, Lamperti ED, Berkovic S, Scheffer I, Cooper EC, Dobyns WB, Minnerath SR, Ross ME, Walsh CA. 1998. Doublecortin, a brainspecific gene mutated in human X-linked lissencephaly and double cortex syndrome, encodes a putative signaling protein. Cell 92(1):63-72. 
Guillonneau X, Piriev NI, Danciger M, Kozak CA, Cideciyan AV, Jacobson SG, Farber DB. 1999. A nonsense mutation in a novel gene is associated with retinitis pigmentosa in a family linked to the RP1 locus. Hum Mol Genet 8(8):1541-6.

Hartong DT, Berson EL, Dryja TP. 2006. Retinitis pigmentosa. Lancet 368(9549):1795809.

Jacobson SG, Cideciyan AV, Iannaccone A, Weleber RG, Fishman GA, Maguire AM, Affatigato LM, Bennett J, Pierce EA, Danciger M, Farber DB, Stone EM. 2000. Disease expression of RP1 mutations causing autosomal dominant retinitis pigmentosa. Invest Ophthalmol Vis Sci 41(7):1898-908.

Kawamura M, Wada Y, Noda Y, Itabashi T, Ogawa S, Sato H, Tanaka K, Ishibashi T, Tamai M. 2004. Novel 2336-2337delCT mutation in RP1 gene in a Japanese family with autosomal dominant retinitis pigmentosa. Am J Ophthalmol 137(6):1137-9.

Khaliq S, Abid A, Ismail M, Hameed A, Mohyuddin A, Lall P, Aziz A, Anwar K, Mehdi SQ. 2005. Novel association of RP1 gene mutations with autosomal recessive retinitis pigmentosa. J Med Genet 42(5):436-8.

Kim MH, Derewenda U, Devedjiev Y, Dauter Z, Derewenda ZS. 2003. Purification and crystallization of the N-terminal domain from the human doublecortin-like kinase. Acta Crystallogr D Biol Crystallogr 59(Pt 3):502-5.

Liu Q, Zhou J, Daiger SP, Farber DB, Heckenlively JR, Smith JE, Sullivan LS, Zuo J, Milam AH, Pierce EA. 2002. Identification and subcellular localization of the RP1 protein in human and mouse photoreceptors. Invest Ophthalmol Vis Sci 43(1):22-32.

Liu Q, Lyubarsky A, Skalet JH, Pugh EN, Jr., Pierce EA. 2003. RP1 is required for the correct stacking of outer segment discs. Invest Ophthalmol Vis Sci 44(10):417183.

Liu Q, Saveliev A, Pierce EA. 2009. The severity of retinal degeneration in Rp1h genetargeted mice is dependent on genetic background. Invest Ophthalmol Vis Sci 50(4):1566-74.

McGee TL, Devoto M, Ott J, Berson EL, Dryja TP. 1997. Evidence that the penetrance of mutations at the RP11 locus causing dominant retinitis pigmentosa is influenced by a gene linked to the homologous RP11 allele. Am J Hum Genet 61(5):1059-66.

Mukhopadhyay R, Holder GE, Moore AT, Webster AR. 2011. Unilateral Retinitis Pigmentosa Occurring in an Individual With a Germline Mutation in the RP1 Gene. Arch Ophthalmol 129(7):954-6.

Payne A, Vithana E, Khaliq S, Hameed A, Deller J, Abu-Safieh L, Kermani S, Leroy BP, Mehdi SQ, Moore AT, Bird AC, Bhattacharya SS. 2000. RP1 protein truncating mutations predominate at the RP1 adRP locus. Invest Ophthalmol Vis Sci 41(13):4069-73.

Pierce EA, Quinn T, Meehan T, McGee TL, Berson EL, Dryja TP. 1999. Mutations in a gene encoding a new oxygen-regulated photoreceptor protein cause dominant retinitis pigmentosa. Nat Genet 22(3):248-54. 
Pierce EA, Saveliev A, Collin RWJ, van den Born I, Liu Q. Mutations in RP1 cause dominant retinitis pigmentosa via a dominant-negative mechanism.; 2010; Washington, USA.

Riazuddin SA, Zulfiqar F, Zhang Q, Sergeev YV, Qazi ZA, Husnain T, Caruso R, Riazuddin S, Sieving PA, Hejtmancik JF. 2005. Autosomal recessive retinitis pigmentosa is associated with mutations in RP1 in three consanguineous Pakistani families. Invest Ophthalmol Vis Sci 46(7):2264-70.

Rivolta C, McGee TL, Rio Frio T, Jensen RV, Berson EL, Dryja TP. 2006. Variation in retinitis pigmentosa-11 (PRPF31 or RP11) gene expression between symptomatic and asymptomatic patients with dominant RP11 mutations. Hum Mutat 27(7):644-53.

Roberts L, Bartmann L, Ramesar R, Greenberg J. 2006. Novel variants in the hotspot region of RP1 in South African patients with retinitis pigmentosa. Mol Vis 12:177-83.

Schwartz SB, Aleman TS, Cideciyan AV, Swaroop A, Jacobson SG, Stone EM. 2003. De novo mutation in the RP1 gene (Arg677ter) associated with retinitis pigmentosa. Invest Ophthalmol Vis Sci 44(8):3593-7.

Singh HP, Jalali S, Narayanan R, Kannabiran C. 2009. Genetic analysis of Indian families with autosomal recessive retinitis pigmentosa by homozygosity screening. Invest Ophthalmol Vis Sci 50(9):4065-71.

Sohocki MM, Daiger SP, Bowne SJ, Rodriquez JA, Northrup H, Heckenlively JR, Birch DG, Mintz-Hittner H, Ruiz RS, Lewis RA, Saperstein DA, Sullivan LS. 2001. Prevalence of mutations causing retinitis pigmentosa and other inherited retinopathies. Hum Mutat 17(1):42-51.

Sullivan LS, Heckenlively JR, Bowne SJ, Zuo J, Hide WA, Gal A, Denton M, Inglehearn CF, Blanton SH, Daiger SP. 1999. Mutations in a novel retina-specific gene cause autosomal dominant retinitis pigmentosa. Nat Genet 22(3):255-9.

Sullivan LS, Bowne SJ, Birch DG, Hughbanks-Wheaton D, Heckenlively JR, Lewis RA, Garcia CA, Ruiz RS, Blanton SH, Northrup H, Gire AI, Seaman R, Duzkale H, Spellicy CJ, Zhu J, Shankar SP, Daiger SP. 2006. Prevalence of disease-causing mutations in families with autosomal dominant retinitis pigmentosa: a screen of known genes in 200 families. Invest Ophthalmol Vis Sci 47(7):3052-64.

Vaclavik V, Gaillard MC, Tiab L, Schorderet DF, Munier FL. 2010. Variable phenotypic expressivity in a Swiss family with autosomal dominant retinitis pigmentosa due to a T494M mutation in the PRPF3 gene. Mol Vis 16:467-75.

Vithana EN, Abu-Safieh L, Pelosini L, Winchester E, Hornan D, Bird AC, Hunt DM, Bustin SA, Bhattacharya SS. 2003. Expression of PRPF31 mRNA in patients with autosomal dominant retinitis pigmentosa: a molecular clue for incomplete penetrance? Invest Ophthalmol Vis Sci 44(10):4204-9.

Won J, Shi LY, Hicks W, Wang J, Hurd R, Naggert JK, Chang B, Nishina PM. 2011. Mouse model resources for vision research. J Ophthalmol 2011:391384.

Xiaoli Z, Weiling F, Pang CP, Yeung KY. 2002. Screening for mutations in a novel retinal-specific gene among Chinese patients with retinitis pigmentosa. Chin Med Sci J 17(4):225-30. 
Zhang X, Chen LJ, Law JP, Lai TY, Chiang SW, Tam PO, Chu KY, Wang N, Zhang M, Pang CP. 2010. Differential pattern of RP1 mutations in retinitis pigmentosa. Mol Vis $16: 1353-60$.

Ziviello C, Simonelli F, Testa F, Anastasi M, Marzoli SB, Falsini B, Ghiglione D, Macaluso C, Manitto MP, Garre C, Ciccodicola A, Rinaldi E, Banfi S. 2005. Molecular genetics of autosomal dominant retinitis pigmentosa (ADRP): a comprehensive study of 43 Italian families. J Med Genet 42(7):e47. 
Table 1: Previously described and novel RP1 mutations leading to ar or adRP.

\begin{tabular}{|c|c|c|c|c|}
\hline Disorder & Exon & Nucleotide Exchange & Protein Effect & Literature \\
\hline $\begin{array}{l}\text { non } \\
\text { syndromic } \\
\text { RP } \\
\end{array}$ & 2 & c.5_6delGT & p.Ser2ArgfsX16 & (Chen et al. 2010) \\
\hline arRP & 3 & c.686delC & p.Pro229GlnX35 & $\begin{array}{l}\text { (Pierce et al. } \\
\text { 2010) }\end{array}$ \\
\hline $\begin{array}{l}\operatorname{arRP} \\
\text { but not } \\
\text { adRP! }\end{array}$ & 4 & c. $1118 \mathrm{C}>\mathrm{T}$ & p.Thr373Ile & $\begin{array}{l}\text { (Payne et al. } \\
\text { 2000; Berson et } \\
\text { al. 2001; Khaliq } \\
\text { et al. 2005) }\end{array}$ \\
\hline arRP & 4 & c.1458_1461dup & p.Glu488X & $\begin{array}{l}\text { (Khaliq et al. } \\
\text { 2005; Riazuddin } \\
\text { et al. 2005) }\end{array}$ \\
\hline $\operatorname{adRP}$ & 4 & c.1498_1499insGT & p.Met500SerfsX33 & $\begin{array}{l}\text { (Payne et al. } \\
\text { 2000) }\end{array}$ \\
\hline adRP & 4 & $\begin{array}{l}\text { 1-bp insertion (A) not clear where } \\
\text { exactly }\end{array}$ & $\begin{array}{l}\text { p.Pro658HisfsX4 } \\
\text { previously: frameshift in } \\
\text { codon p.Pro } 658\end{array}$ & $\begin{array}{l}\text { (Jacobson et al. } \\
2000)\end{array}$ \\
\hline $\begin{array}{l}\text { RP } \\
\text { sporadic } \\
\text { case }\end{array}$ & 4 & c. $2005 \mathrm{G}>\mathrm{A}$ & p.Ala669Thr & $\begin{array}{l}\text { (Khaliq et al. } \\
\text { 2005) }\end{array}$ \\
\hline $\operatorname{adRP}$ & 4 & c. $2025 \mathrm{del} A$ & $\begin{array}{l}\text { p.Lys } 675 \text { AsnfsX7 } \\
\text { incomplete penetrance or } \\
\text { later age of onset }\end{array}$ & $\begin{array}{l}\text { This } r \text { study: } \\
\text { CIC00864, F556 } \\
\text { and CIC03407, } \\
\text { F1452 }\end{array}$ \\
\hline
\end{tabular}




\begin{tabular}{|c|c|c|c|c|}
\hline $\begin{array}{l}\text { adRP } \\
\text { sporadic } \\
\text { case } \\
\text { novo }\end{array}$ & 4 & c. $2029 \mathrm{C}>\mathrm{T}$ & $\begin{array}{l}\text { p.Arg677X } \\
\text { incomplete penetrance }\end{array}$ & $\begin{array}{l}\text { (Bowne et al. } \\
\text { 1999; } \\
\text { Guillonneau et al. } \\
\text { 1999; Pierce et al. } \\
\text { 1999; Sullivan et } \\
\text { al. 1999; } \\
\text { Jacobson et al. } \\
\text { 2000; Payne et al. } \\
\text { 2000; Baum et al. } \\
\text { 2001; Berson et } \\
\text { al. 2001; Sohocki } \\
\text { et al. 2001; Xiaoli } \\
\text { et al. 2002; } \\
\text { Schwartz et al. } \\
\text { 2003; Ziviello et } \\
\text { al. 2005; Chiang } \\
\text { et al. 2006; } \\
\text { Gamundi et al. } \\
\text { 2006; Roberts et } \\
\text { al. 2006; Sullivan } \\
\text { et al. 2006) }\end{array}$ \\
\hline adRP & 4 & c.2029delC & p.Arg677AspfsX5 & $\begin{array}{l}\text { (Bowne et al. } \\
1999)\end{array}$ \\
\hline adRP & 4 & $\begin{array}{l}\text { c. } 2035 \mathrm{C}>\mathrm{T} \\
\text { in } c i s \text { with c.5377C }>\mathrm{T}\end{array}$ & $\begin{array}{l}\text { p.Gln679X } \\
\text { in cis with p.Pro1793Ser }\end{array}$ & $\begin{array}{l}\text { (Sullivan et al. } \\
\text { 1999; Berson et } \\
\text { al. 2001) }\end{array}$ \\
\hline adRP & 4 & c. $2056 \mathrm{C}>\mathrm{T}$ & p.Gln686X & $\begin{array}{l}\text { (Gamundi et al. } \\
\text { 2006) }\end{array}$ \\
\hline adRP & 4 & c. $2065 \mathrm{C}>\mathrm{T}$ & p.Gln689X & $\begin{array}{l}\text { This study: } \\
\text { CIC01529, F1332 }\end{array}$ \\
\hline
\end{tabular}




\begin{tabular}{|c|c|c|c|c|}
\hline adRP & 4 & c. $2098 \mathrm{G}>\mathrm{T}$ & p.Glu700X & $\begin{array}{l}\text { (Bowne et al. } \\
\text { 1999; Payne et al. } \\
\text { 2000) }\end{array}$ \\
\hline adRP4 & 4 & c. $2115 \operatorname{del} A$ & $\begin{array}{l}\text { p.Gly706ValsfsX7 } \\
\text { previously p.Lys705fsX712 }\end{array}$ & $\begin{array}{l}\text { (Gamundi et al. } \\
\text { 2006) }\end{array}$ \\
\hline adRP & 4 & c.2164_2165delinsG & $\begin{array}{l}\text { p.Lys } 722 \text { GlufsX16 } \\
\text { previously p.Lys722fsX737 }\end{array}$ & $\begin{array}{l}\text { (Gamundi et al. } \\
\text { 2006) }\end{array}$ \\
\hline adRP & 4 & c. $2167 \mathrm{G}>\mathrm{T}$ & p.Gly723X & $\begin{array}{l}\text { (Berson et al. } \\
\text { 2001; Sohocki et } \\
\text { al. 2001; Sullivan } \\
\text { et al. 2006) }\end{array}$ \\
\hline adRP & 4 & c.2168_2181del & p.Ile725ArgfsX6 & $\begin{array}{l}\text { (Bowne et al. } \\
\text { 1999; Payne et al. } \\
\text { 2000) }\end{array}$ \\
\hline $\operatorname{adRP}$ & 4 & c. $2169 \mathrm{delA}$ & p.Ile725TyrfsX13 & $\begin{array}{l}\text { This study: } \\
\text { CIC0009, F8 and } \\
\text { CIC02805, F1039 }\end{array}$ \\
\hline adRP & 4 & $\begin{array}{l}\text { c.2172dupG } \\
\text { Previously c.2169_2170insG }\end{array}$ & p.Ile725AspfsX4 & $\begin{array}{l}\text { (Bowne et al. } \\
\text { 1999; Sohocki et } \\
\text { al. 2001) }\end{array}$ \\
\hline adRP & 4 & c.2171_2186del & p.Gly724GlufsX9 & $\begin{array}{l}\text { (Payne et al. } \\
\text { 2000) }\end{array}$ \\
\hline adRP & 4 & c. $2185 \mathrm{delG}$ & p. Glu729LysfsX9 & $\begin{array}{l}\text { (Berson et al. } \\
2001)\end{array}$ \\
\hline adRP & 4 & c.2206_2207insT & p.Thr736IlefsX4 & $\begin{array}{l}\text { (Payne et al. } \\
\text { 2000) }\end{array}$ \\
\hline adRP & 4 & c. $2232 \mathrm{~T}>\mathrm{A}$ & p.Cys744X & $\begin{array}{l}\text { (Bowne et al. } \\
\text { 1999; Payne et al. } \\
\text { 2000) }\end{array}$ \\
\hline
\end{tabular}




\begin{tabular}{|c|c|c|c|c|}
\hline adRP & 4 & c. $2239 \mathrm{delA}$ & p.Ser747ValfsX16 & $\begin{array}{l}\text { (Jacobson et al. } \\
2000)\end{array}$ \\
\hline adRP & 4 & c. $2275 \mathrm{~A}>\mathrm{T}$ & p.Arg759X & $\begin{array}{l}\text { This study: } \\
\text { CIC01239, F749 }\end{array}$ \\
\hline adRP & 4 & c.2284_2289del & $\begin{array}{l}\text { p.Leu762_Asn763del } \\
\text { previously mentioned } \\
\text { frameshift with premature } \\
\text { X? }\end{array}$ & $\begin{array}{l}\text { (Payne et al. } \\
2000)\end{array}$ \\
\hline adRP & 4 & $\begin{array}{l}\text { c.2285_2289del } \\
\text { previously c.2280_2284del }\end{array}$ & Leu762TyrfsX17 & $\begin{array}{l}\text { (Bowne et al. } \\
\text { 1999; Pierce et al. } \\
\text { 1999; Jacobson et } \\
\text { al. 2000; Payne et } \\
\text { al. 2000; Berson } \\
\text { et al. 2001; } \\
\text { Sohocki et al. } \\
\text { 2001; Sullivan et } \\
\text { al. 2006) }\end{array}$ \\
\hline adRP & 4 & c.2287_2290del & p.Asn763LeufsX11 & $\begin{array}{l}\text { (Pierce et al. } \\
\text { 1999; Berson et } \\
\text { al. 2001) }\end{array}$ \\
\hline adRP & 4 & $\begin{array}{l}\text { c.2304delC } \\
\text { previously c.2303delC }\end{array}$ & p.Lys769ArgfsX6 & $\begin{array}{l}\text { (Bowne et al. } \\
\text { 1999; Sohocki et } \\
\text { al. 2001) }\end{array}$ \\
\hline adRP & 4 & c. $2332 \mathrm{~A}>\mathrm{T}$ & $\begin{array}{l}\text { p.Lys } 778 \mathrm{X} \\
\text { incomplete penetrence }\end{array}$ & $\begin{array}{l}\text { (Dietrich et al. } \\
\text { 2002) }\end{array}$ \\
\hline adRP & 4 & c.2336_2337delCT & p.Ser779X & $\begin{array}{l}\text { (Kawamura et al. } \\
\text { 2004) }\end{array}$ \\
\hline $\operatorname{adRP}$ & 4 & c.2590_2599del & $\begin{array}{l}\text { p.Ile864LysfsX11 } \\
\text { incomplete penetrence }\end{array}$ & $\begin{array}{l}\text { (Roberts et al. } \\
\text { 2006) }\end{array}$ \\
\hline
\end{tabular}




\begin{tabular}{|c|c|c|c|c|}
\hline adRP & 4 & c.2594_2596del & p.Thr865_Leu866delinsIle & $\begin{array}{l}\text { (Payne et al. } \\
2000)\end{array}$ \\
\hline adRP & 4 & $\begin{array}{l}\text { c.2613dupA } \\
\text { previously c.2608_2609insA }\end{array}$ & p.Arg872ThrfsX2 & $\begin{array}{l}\text { (Payne et al. } \\
2000)\end{array}$ \\
\hline adRP & 4 & c. $2732 \mathrm{C}>\mathrm{A}$ & $\begin{array}{l}\text { p.Ser911X } \\
\text { incomplete penetrence }\end{array}$ & $\begin{array}{lll}\text { (Roberts et al. } \\
2006)\end{array}$ \\
\hline $\operatorname{arRP}$ & 4 & c.2847delT & p.Asn949LysfsX32 & $\begin{array}{l}\text { (Singh et al. } \\
2009)\end{array}$ \\
\hline $\operatorname{adRP}$ & 4 & c. $2951 \mathrm{~A}>\mathrm{G}$ & $\begin{array}{l}\text { p.Asp984Gly } \\
\text { phenoytpe variations in one } \\
\text { family }\end{array}$ & $\begin{array}{l}\text { (Chiang et al. } \\
\text { 2006) }\end{array}$ \\
\hline adRP & 4 & c. $3157 \mathrm{delT}$ & p.Tyr1053ThrfsX4 & $\begin{array}{l}\text { (Jacobson et al. } \\
2000)\end{array}$ \\
\hline Simplex RP & 4 & c. $4108 \mathrm{~A}>\mathrm{G}$ & p.Lys1370Glu & $\begin{array}{l}\text { (Zhang et al. } \\
2010)\end{array}$ \\
\hline $\operatorname{arRP}$ & 4 & $\begin{array}{l}\text { c. } 4555 \mathrm{delA} \\
\text { previously c. } 4703 \mathrm{delA}\end{array}$ & $\begin{array}{l}\text { p.Arg1519GlufsX2 } \\
\text { previously } \\
\text { p.Arg1519fsX1521 }\end{array}$ & $\begin{array}{l}\text { (Riazuddin et al. } \\
\text { 2005) }\end{array}$ \\
\hline $\begin{array}{l}\text { non } \\
\text { syndromic } \\
\text { RP }\end{array}$ & 4 & $\begin{array}{l}\text { c.4941dupT } \\
\text { previously c.4941_4942insT }\end{array}$ & p.Pro1648SerfsX13 & (Chen et al. 2010) \\
\hline Simplex RP & 4 & c. $4955 \mathrm{G}>\mathrm{T}$ & p.Arg1652Leu & $\begin{array}{l}\text { (Zhang et al. } \\
2005)\end{array}$ \\
\hline $\operatorname{arRP}$ & 4 & $\begin{array}{l}\text { c. } 5252 \mathrm{delA} \\
\text { previously c.5400delA }\end{array}$ & $\begin{array}{l}\text { p.Asn1751IlefsX4 } \\
\text { previously } \\
\text { p.Asn1751fsX1754 }\end{array}$ & $\begin{array}{l}\text { (Riazuddin et al. } \\
\text { 2005) }\end{array}$ \\
\hline
\end{tabular}


Nucleotide numbering reflects cDNA numbering with +1 corresponding to the A of the ATG translation initiation codon in the reference sequence NM_006269.1, according to journal guidelines (www.hgvs.org/mutnomen). The initiation codon is codon 1 .

Table 2: Clinical data of affected index patients from families with adRP due to $R 1$ mutations

\begin{tabular}{|c|c|c|c|c|c|c|c|c|c|c|c|c|c|}
\hline Patient & Family & Mutation & $\begin{array}{l}\text { Age at } \\
\text { time of } \\
\text { testing }\end{array}$ & $\begin{array}{c}\text { Age at } \\
\text { time of } \\
\text { diagnosis }\end{array}$ & Sex & $\begin{array}{c}\text { Relevant medical } \\
\text { and } \\
\text { ophthalmology } \\
\text { history }\end{array}$ & \begin{tabular}{|c|} 
Family history \\
of incomplete \\
penetrance or \\
variable \\
expressivity
\end{tabular} & $\begin{array}{c}\text { Symptoms at } \\
\text { time of } \\
\text { diagnosis }\end{array}$ & $\begin{array}{c}\text { BCVA } \\
\text { OD/OS } \\
\text { Refraction }\end{array}$ & Lens & $\begin{array}{c}\text { Fundus } \\
\text { examination }\end{array}$ & OCT & AF imaging \\
\hline CIC009 & $\begin{array}{l}8 \\
\text { (rest of the } \\
\text { family } \\
\text { reported } \\
\text { elsewhere) }\end{array}$ & $\begin{array}{c}\text { c.2169delA } \\
\text { p.Ile725TyrfsX13 }\end{array}$ & 70 & 15 & M & $\begin{array}{c}\text { Trabeculectomy } \\
\text { Bilateral cataract } \\
\text { surgery age } 60\end{array}$ & $\begin{array}{l}\text { Incomplete } \\
\text { penetrrance }\end{array}$ & $\begin{array}{l}\text { Night vision } \\
\text { problems }\end{array}$ & $\begin{array}{c}20 / 125 \\
20 / 32 \\
-5.75(- \\
0.75) 90^{\circ} \\
-6(-1) 145^{\circ}\end{array}$ & $\begin{array}{l}\text { Bilateral } \\
\text { IOL }\end{array}$ & $\begin{array}{l}\text { Narrowed blood } \\
\text { vessels, pale optic } \\
\text { discs, RPE } \\
\text { changes in the } \\
\text { periphery, } \\
\text { perifoveal atrophy } \\
\text { OD }\end{array}$ & $\begin{array}{l}\text { Foveal thinning } \\
\text { OD; relative } \\
\text { preservation of } \\
\text { foveal lamination } \\
\text { OS }\end{array}$ & $\begin{array}{l}\text { Patchy loss of AF } \\
\text { outside the } \\
\text { vascular arcades; } \\
\text { perifoveal loss of } \\
\text { AF OD }\end{array}$ \\
\hline CIC00864 & 556 & $\begin{array}{c}\text { c.2025delA } \\
\text { p.Lys675AsnfsX7 }\end{array}$ & 48 & 25 & F & None & $\begin{array}{c}\text { Possible } \\
\text { incomplete } \\
\text { penetrance with } \\
\text { asymptomatic } \\
\text { 19-year old } \\
\text { daughter and } \\
\text { variable } \\
\text { expressivity } \\
\text { with father } \\
\text { diagnosed at } \\
\text { age 55 }\end{array}$ & $\begin{array}{l}\text { Initially } \\
\text { decrease visual } \\
\text { field and mild } \\
\text { night vision } \\
\text { problem }\end{array}$ & $\begin{array}{c}20 / 25 \\
20 / 40 \\
-5.75(-1) 60^{\circ} \\
-5.75(-1) 150^{\circ}\end{array}$ & $\begin{array}{c}\text { Bilateral } \\
\text { posterior } \\
\text { subcapsular } \\
\text { cataract }\end{array}$ & $\begin{array}{l}\text { Narrowed blood } \\
\text { vessels, relatively } \\
\text { well colored optic } \\
\text { nerve discs and } \\
\text { bone spicule } \\
\text { pigment migration } \\
\text { in the periphery } \\
\text { and relatively } \\
\text { spared macula }\end{array}$ & $\begin{array}{l}\text { Preservation of } \\
\text { foveal lamination }\end{array}$ & $\begin{array}{c}\text { Low quality due to } \\
\text { cataract } \\
\text { Normal macular } \\
\text { autofluorescence }\end{array}$ \\
\hline CIC01234 & 745 & $\begin{array}{l}\text { Uncertain } \\
\text { pathogenic } \\
\text { variant: } \\
\text { c.4193C>G } \\
\text { p.Ser1398 }\end{array}$ & 46 & 6 & $\mathrm{~F}$ & $\begin{array}{c}\text { Pneumothorax } \\
\text { Bilateral cataract } \\
\text { surgery age } 44\end{array}$ & Not reported & $\begin{array}{l}\text { No symptoms at } \\
\text { diagnosis } \\
\text { (family } \\
\text { investigation) }\end{array}$ & $\begin{array}{c}20 / 200 \\
20 / 160 \\
+2.50 \\
+1.50(- \\
0.50) 170^{\circ}\end{array}$ & $\begin{array}{l}\text { Bilateral } \\
\text { IOL }\end{array}$ & $\begin{array}{c}\text { Narrowed blood } \\
\text { vessels, pale optic } \\
\text { nerve disc, } \\
\text { widespread RPE } \\
\text { changes in the } \\
\text { periphery and } \\
\text { macular atrophy }\end{array}$ & $\begin{array}{l}\text { Macular thinning } \\
\text { with loss of ONL }\end{array}$ & $\begin{array}{c}\text { Loss of AF outside } \\
\text { the arcades and } \\
\text { within the foveal } \\
\text { region }\end{array}$ \\
\hline CIC01239 & 749 & & 60 & 25 & M & $\begin{array}{l}\text { Bilateral cataract } \\
\text { surgery age } 45\end{array}$ & Not reported & $\begin{array}{c}\text { Night vision } \\
\text { problems } \\
\text { Mainly bothered }\end{array}$ & $\begin{array}{c}20 / 25 \\
20 / 40 \\
-2.50(-\end{array}$ & $\begin{array}{l}\text { Bilateral } \\
\text { IOL }\end{array}$ & $\begin{array}{l}\text { Narrowed blood } \\
\text { vessels, relatively } \\
\text { well colored optic }\end{array}$ & $\begin{array}{l}\text { OD Preservation } \\
\text { of foveal } \\
\text { lamination }\end{array}$ & $\begin{array}{l}\text { Patchy loss of AF } \\
\text { along the vascular } \\
\text { arcades; OD }\end{array}$ \\
\hline
\end{tabular}




\begin{tabular}{|c|c|c|c|c|c|c|c|c|c|c|c|c|c|}
\hline & & $\begin{array}{l}\text { c. } 2275 A>T \\
\text { p.Arg759X }\end{array}$ & & & & & & $\begin{array}{l}\text { at age } 55 \text { by } \\
\text { constricted } \\
\text { visual field }\end{array}$ & $\begin{array}{c}0.50) 95^{\circ} \\
-5(-0.50) 90^{\circ}\end{array}$ & & $\begin{array}{l}\text { nerve discs, little } \\
\text { pigment migration } \\
\text { in the periphery; } \\
\text { OD relatively } \\
\text { spared macula. OS } \\
\text { CME }\end{array}$ & OS CME & $\begin{array}{l}\text { Normal macular } \\
\text { autofluorescence; } \\
\text { OS perifoveol loss } \\
\text { of AF on the } \\
\text { temporal side and } \\
\text { abnormal foveal af } \\
\text { related to the CMO }\end{array}$ \\
\hline CIC02805 & 1039 & $\begin{array}{c}\text { c.2169delA } \\
\text { p.Ile725TyrfsX13 }\end{array}$ & 58 & 30 & $\mathrm{M}$ & None & $\begin{array}{c}\text { Variable age of } \\
\text { onset }\end{array}$ & $\begin{array}{l}\text { Night vision } \\
\text { problem } \\
\text { Decreased } \\
\text { vision }\end{array}$ & $\begin{array}{c}20 / 40 \\
20 / 32 \\
-2.25(- \\
0.75) 90^{\circ} \\
-2.75(- \\
0.50) 90)\end{array}$ & $\begin{array}{c}\text { Bilateral } \\
\text { posterior } \\
\text { subcapsular } \\
\text { cataract }\end{array}$ & $\begin{array}{l}\text { Narrowed blood } \\
\text { vessels, pale optic } \\
\text { discs, little RPE } \\
\text { changes in the } \\
\text { periphery }\end{array}$ & $\begin{array}{c}\text { Relative } \\
\text { conservation of } \\
\text { foveal lamination }\end{array}$ & $\begin{array}{l}\text { Patchy loss of AF } \\
\text { outside the } \\
\text { vascular arcades } \\
\text { and some } \\
\text { perifoveal loss }\end{array}$ \\
\hline CIC01529 & 1332 & $\begin{array}{l}\text { c. } 2065 C>T \\
\text { p.Gln689X }\end{array}$ & 51 & 30 & M & None & $\begin{array}{l}\text { Later age of } \\
\text { onset reported } \\
\text { in other family } \\
\text { members }\end{array}$ & $\begin{array}{l}\text { Night vision } \\
\text { problem }\end{array}$ & $\begin{array}{c}20 / 32 \\
20 / 25 \\
-2.50(- \\
1.25) 20^{\circ} \\
-3(-1) 140^{\circ}\end{array}$ & \begin{tabular}{|c} 
Mild \\
bilateral \\
posterior \\
subcapsular \\
cataract
\end{tabular} & $\begin{array}{l}\text { Narrowed blood } \\
\text { vessels, pale optic } \\
\text { nerve discs, RPE } \\
\text { changes in the } \\
\text { periphery and in } \\
\text { perifoveal region }\end{array}$ & $\begin{array}{c}\text { Relative } \\
\text { conservation of } \\
\text { foveal lamination }\end{array}$ & $\begin{array}{l}\text { Patchy loss of AF } \\
\text { outside the } \\
\text { vascular arcades } \\
\text { and in the } \\
\text { perifoveal area }\end{array}$ \\
\hline CIC03407 & 1452 & $\begin{array}{c}\text { c.2025delA } \\
\text { p.Lys675AsnfsX7 }\end{array}$ & 48 & 27 & $\mathrm{~F}$ & Deafness & Not reported & $\begin{array}{l}\text { Night vision } \\
\text { problem }\end{array}$ & $\begin{array}{c}20 / 200 \\
20 / 800 \\
\text { Plano } \\
-0.25(- \\
0.50) 130^{\circ}\end{array}$ & \begin{tabular}{|} 
Significant \\
bilateral \\
posterior \\
subcapsular \\
cataract \\
OD $>$ OS
\end{tabular} & $\begin{array}{l}\text { Narrowed blood } \\
\text { vessels, relatively } \\
\text { well coloured } \\
\text { optic nerve heads, } \\
\text { little RPE changes } \\
\text { in the periphery, } \\
\text { macular atrophic } \\
\text { changes }\end{array}$ & $\begin{array}{l}\text { Macular thinning } \\
\text { with loss of ONL }\end{array}$ & $\begin{array}{l}\text { Patchy loss of AF } \\
\text { in periphery and } \\
\text { foveal region }\end{array}$ \\
\hline
\end{tabular}

BCVA: best corrected visual acuity; OD: Oculis dextra (right eye); OS: Oculis Sinistra (left eye); OCT: Optical Coherence Tomography; AF: autofluorescence; IOL: intra ocular lens; CME: cystoid macular edema; RPE: retinal pigment epithelium; ONL: Outer Nuclear Layer

Nucleotide numbering reflects cDNA numbering with +1 corresponding to the A of the ATG translation initiation codon in the reference sequence NM_006269.1, according to journal guidelines (www.hgvs.org/mutnomen). The initiation codon is codon 1. 
Table 3: Function data

\begin{tabular}{|c|c|c|c|c|c|}
\hline Patient & Mutation & $\begin{array}{c}\text { Colour vision } \\
\text { (15 desaturated Hue) }\end{array}$ & $\begin{array}{c}\text { Binocular Goldman visual field, III4 } \\
\text { isopter }\end{array}$ & $\begin{array}{c}\text { Full field } \\
\text { ERG }\end{array}$ & Multifocal ERG \\
\hline CIC0009 & $\begin{array}{c}\text { c.2169delA } \\
\text { p.Ile725TyrfsX13 }\end{array}$ & Dyschromatopsia with tritan axis & $10^{\circ}$ horizontally and vertically & ND & ND \\
\hline CIC00864 & $\begin{array}{c}\text { c. } 2025 \text { delA } \\
\text { p.Lys675AsnfsX7 }\end{array}$ & normal & $35^{\circ}$ horizontally and $30^{\circ}$ vertically & ND & $\begin{array}{c}\text { Only residual responses to central } \\
\text { hexagons }\end{array}$ \\
\hline CIC01234 & $\begin{array}{c}\text { Uncertain pathogenic } \\
\text { variant: } \\
\text { c. } 4193 \mathrm{C}>\mathrm{G} \\
\text { p.Ser1398 }\end{array}$ & $\begin{array}{l}\text { Dyschromatopsia with no specific } \\
\text { axis }\end{array}$ & $40^{\circ}$ horizontally and $20^{\circ}$ vertically & ND & 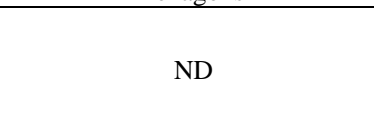 \\
\hline CIC01239 & $\begin{array}{l}\text { c. } 2275 \mathrm{~A}>\mathrm{T} \\
\text { p.Arg759X }\end{array}$ & Few mistakes without axis & $10^{\circ}$ horizontally and vertically & ND & $\begin{array}{c}\text { Only residual responses to central } \\
\text { hexagons }\end{array}$ \\
\hline CIC02805 & $\begin{array}{c}\text { c.2169delA } \\
\text { p.Ile725TyrfsX13 }\end{array}$ & Few mistakes of protan axis & $20^{\circ}$ horizontally and vertically & ND & $\begin{array}{l}\text { Only residual responses to central } \\
\text { hexagons }\end{array}$ \\
\hline CIC01529 & $\begin{array}{l}\text { c. } 2065 C>T \\
\text { p.Gln689X }\end{array}$ & Normal & $10^{\circ}$ horizontally and vertically & ND & $\begin{array}{l}\text { Only residual responses to central } \\
\text { hexagons }\end{array}$ \\
\hline CIC03407 & $\begin{array}{c}\text { c.2025delA } \\
\text { p.Lys675AsnfsX7 }\end{array}$ & Dyschromatopsia of tritan axis & Inferior to 10 central degree & ND & ND \\
\hline
\end{tabular}

ND: not detectable

Nucleotide numbering reflects cDNA numbering with +1 corresponding to the A of the ATG translation initiation codon in the reference sequence NM_006269.1, according to journal guidelines (www.hgvs.org/mutnomen). The initiation codon is codon 1. 


\section{FIGURE LEGENDS}

Figure 1: Fundus autofluorescence and optical coherence appearance in 2 patients from this French cohort with RPI truncating mutations.
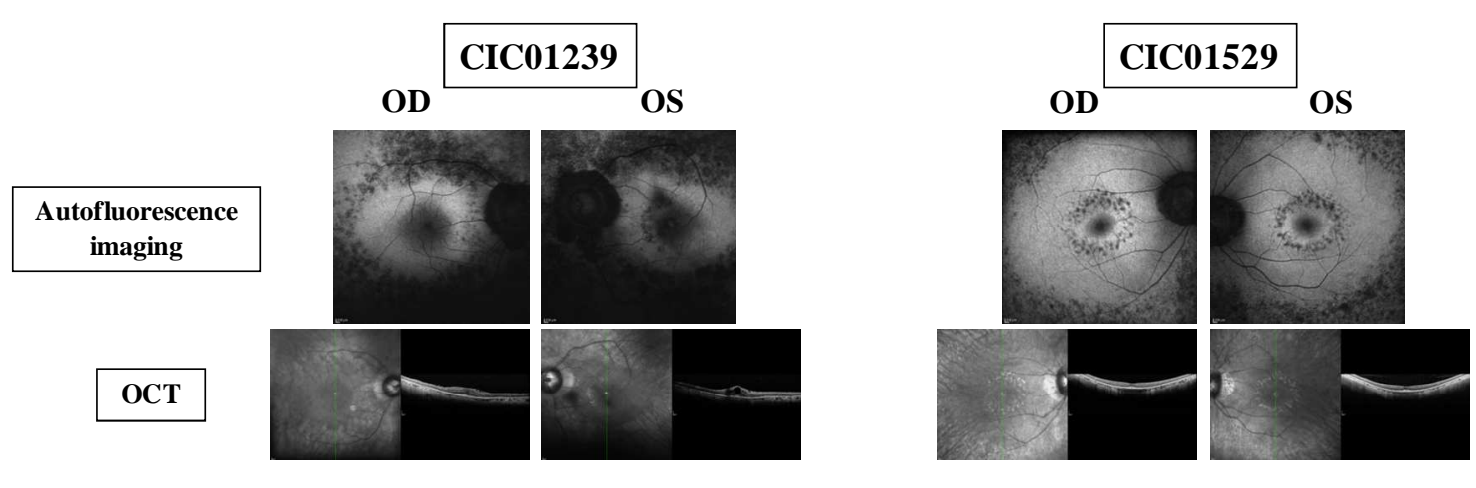


\section{Supplementary data}

Methods

Clinical assessment

Patients with a provisional diagnosis of autosomal dominant rod-cone dystrophy, (adRP) were ascertained in the Clinical Investigating Centre of Quinze-Vingts Hospital. Informed consent was obtained from each patient and normal controls after explanation of the study and its potential outcome. The study protocol adhered to the tenets of the Declaration of Helsinki and was approved by the local ethics committee. Each patient underwent full ophthalmic examination with clinical assessment as described earlier (Audo et al., 2010, RHO mutations). For additional family members who could not come to our centre for examination, ophthalmic records were obtained from local ophthalmologists.

\section{Mutation detection}

Total genomic DNA was extracted from peripheral blood leucocytes according to manufacturer recommendation (Puregen Kit, Qiagen, Courtaboeuf, France). Subsequently, direct genomic sequencing of RP1 (Accession Number: NM_006269.1) was performed. All 4 exons of which exons 2-4 are coding, and flanking intronic regions of RP1 were PCR amplified in 20 fragments (RP1 RefSeq NM_006269.1) using a polymerase (HotFire, Solis Biodyne, Estonia) in the presence of $1.5 \mathrm{mM}$ $\mathrm{MgCl}_{2}$ and at an annealing temperature of $58^{\circ} \mathrm{C}$ (Primers can be obtained by request). The PCR products were enzymatically purified (ExoSAP-IT, USB Corporation, Cleveland, Ohio, USA purchased from GE Healthcare, Orsay, France) and sequenced with a commercially available sequencing mix (BigDyeTerm v1.1 CycleSeq kit, Applied Biosystems, Courtaboeuf, France). The sequenced products were purified on a presoaked Sephadex G-50 (GE Healthcare) 96-well multiscreen filter plate (Millipore, Molsheim, France), the purified product analyzed on an automated 48-capillary sequencer (ABI 3730 Genetic analyzer, Applied Biosystems) and the results interpreted by applying a software (SeqScape, Applied Biosystems). For the missense mutation 190 commercially available control samples were used to validate the pathogenicity (Human random control panel 1-3, Health Protection Agency Culture Collections, Salisbury, United Kingdom). 


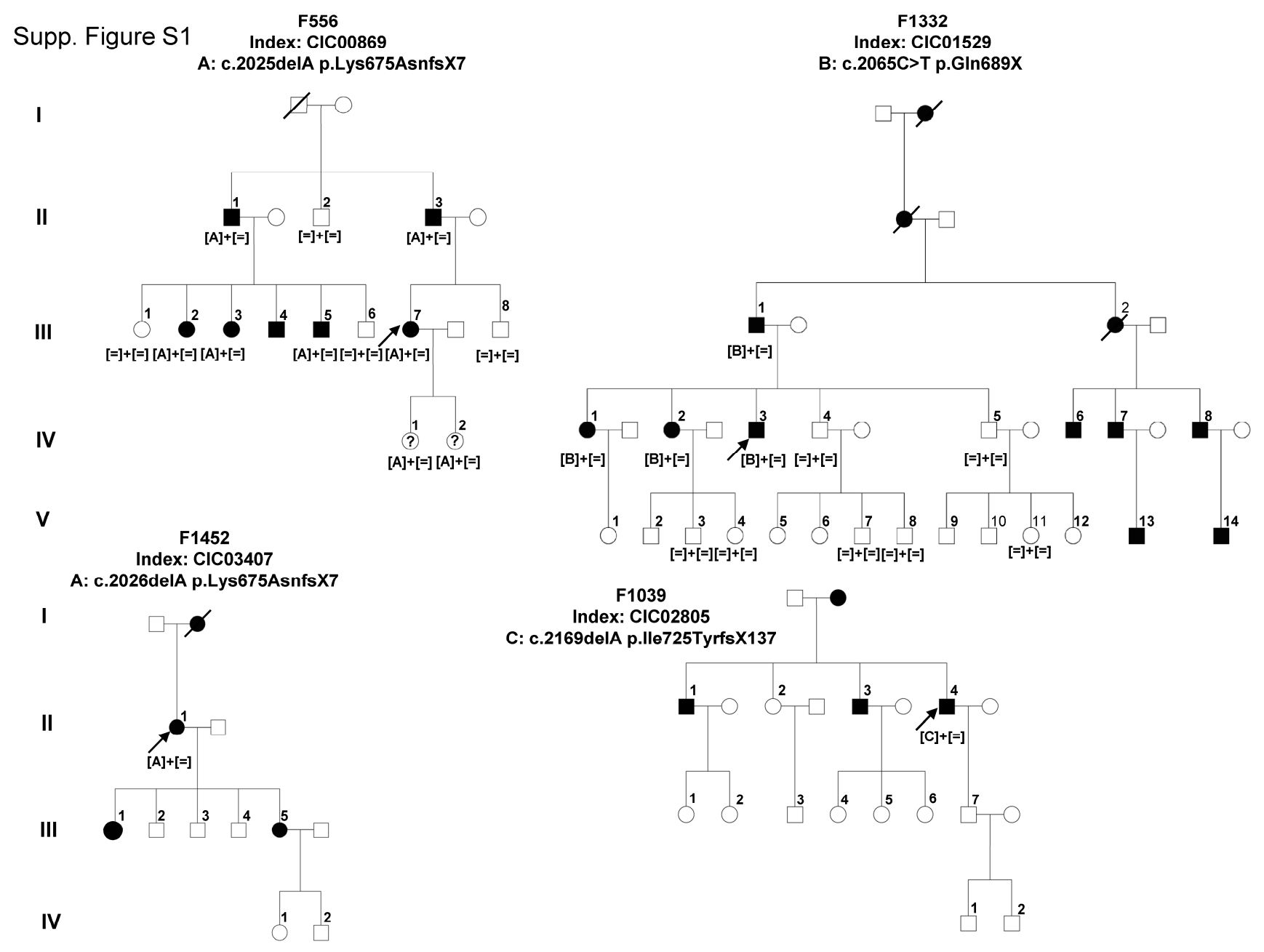


F749

Index: $\mathrm{ClC} 01239$

D: c.2275A $>$ T p.Arg759X

II

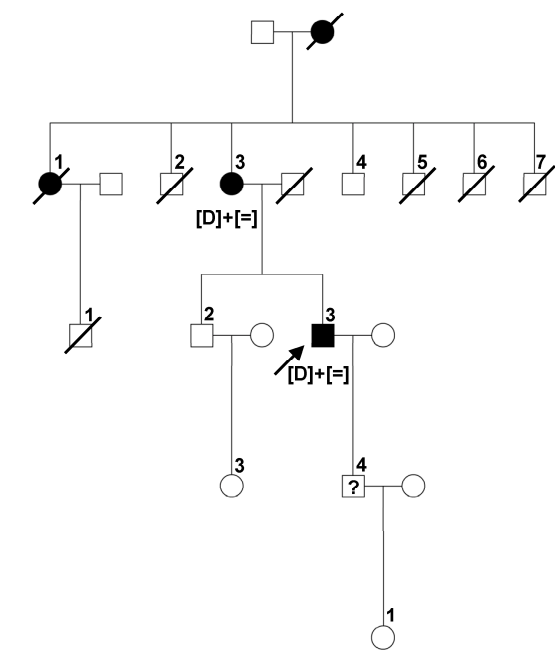

F745

Index: CIC01234

E: c.4193C>G p.Ser1398Lys

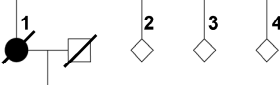

III

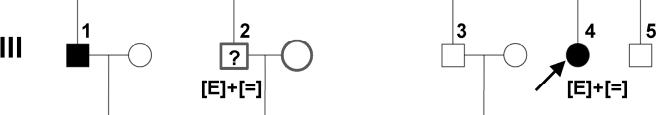

IV

$[=]+[=]$ 
Supp. Figure 1: Pedigrees of families with different novel $R P 1$ mutations and co-segregation in available family members.

Filled symbols represent affected and unfilled unaffected persons. Squares indicate males, circles females. Arrows reflect the index patients. Equation symbols represent unaffected alleles. 
Supplementary Table 1: Novel RP1 mutations in a French adRP cohort.

\begin{tabular}{|c|c|c|c|c|c|c|}
\hline Index (families) & Exon & Nucleotide Exchange & Protein Effect & controls & SIFT & POLYPHEN \\
\hline $\begin{array}{l}\text { CIC00864 } \\
\text { (F556) (III.7) }\end{array}$ & 4 & c. $2025 \mathrm{delA}$ & p.Lys675AsnfsX7 & nd & na & na \\
\hline $\begin{array}{l}\text { CIC00865 (IV.2) } \\
\text { asymptomatic } \\
\text { daughter }\end{array}$ & & c. $2025 \mathrm{delA}$ & p.Lys675AsnfsX7 & & & \\
\hline $\begin{array}{l}\text { CIC04903 (IV.8) } \\
\text { unaffected brother }\end{array}$ & & no & no & & & \\
\hline $\begin{array}{l}\text { CIC04950 (II.1) } \\
\text { affected uncle }\end{array}$ & & c. $2025 \mathrm{delA}$ & p.Lys675AsnfsX7 & & & \\
\hline $\begin{array}{l}\text { CIC04968 (II.2) } \\
\text { unaffected } \\
\text { uncle }\end{array}$ & & no & no & & & \\
\hline $\begin{array}{l}\text { CIC04969 (II.3) } \\
\text { affected father }\end{array}$ & & c. $2025 \mathrm{delA}$ & p.Lys675AsnfsX7 & & & \\
\hline $\begin{array}{l}\text { CIC05003, (III.3) } \\
\text { affected cousin }\end{array}$ & & c. 2025 delA & p.Lys675AsnfsX7 & & & \\
\hline $\begin{array}{l}\text { CIC05043, (III.6) } \\
\text { unaffected cousin }\end{array}$ & & no & no & & & \\
\hline
\end{tabular}




\begin{tabular}{|c|c|c|c|c|c|}
\hline $\begin{array}{l}\text { CIC05051, (III.5) } \\
\text { affected cousin }\end{array}$ & & c. 2025 delA & p.Lys675AsnfsX7 & & \\
\hline $\begin{array}{l}\text { CIC05053, (III.1) } \\
\text { unaffected cousin }\end{array}$ & & no & no & & \\
\hline $\begin{array}{l}\text { CIC05093 (IV.2) } \\
\text { asymptomatic } \\
\text { daughter }\end{array}$ & & c. 2025 delA & p.Lys675AsnfsX7 & & \\
\hline $\begin{array}{l}\text { CIC05168 (III.2) } \\
\text { affected cousin }\end{array}$ & & c. 2025 delA & p.Lys675AsnfsX7 & & \\
\hline $\begin{array}{l}\text { CIC03407 (F1452) } \\
\text { (II.1) }\end{array}$ & 4 & c. 2025 delA & p.Lys675AsnfsX7 nd & na & na \\
\hline $\begin{array}{l}\text { CIC01529 (F1332) } \\
\text { (IV.3) }\end{array}$ & 4 & c. $2065 \mathrm{C}>\mathrm{T}$ & p.Gln689X & na & na \\
\hline $\begin{array}{l}\text { CIC05213 (III.1) } \\
\text { affected father }\end{array}$ & & c. $2065 \mathrm{C}>\mathrm{T}$ & p.Gln689X & & \\
\hline $\begin{array}{l}\text { CIC05179 (IV.1) } \\
\text { affected sister }\end{array}$ & & c. $2065 \mathrm{C}>\mathrm{T}$ & p.Gln689X & & \\
\hline $\begin{array}{l}\text { CIC05185 (IV.2) } \\
\text { affected sister }\end{array}$ & & c. $2065 \mathrm{C}>\mathrm{T}$ & p.Gln689X & & \\
\hline $\begin{array}{l}\text { CIC05312 (IV.4) } \\
\text { unaffected brother }\end{array}$ & & no & no & & \\
\hline
\end{tabular}




\begin{tabular}{|c|c|c|c|c|c|}
\hline $\begin{array}{l}\text { CIC05193 (IV.5) } \\
\text { unaffected brother }\end{array}$ & & no & no & & \\
\hline $\begin{array}{l}\text { CIC05310 (V.3) } \\
\text { unaffected nephew }\end{array}$ & & no & no & & \\
\hline $\begin{array}{l}\text { CIC05186 (V.4) } \\
\text { unaffected niece }\end{array}$ & & no & no & & \\
\hline $\begin{array}{l}\text { CIC05253 (V.7) } \\
\text { unaffected nephew }\end{array}$ & & no & no & & \\
\hline $\begin{array}{l}\text { CIC05311 (V.8) } \\
\text { unaffected nephew }\end{array}$ & & no & no & & \\
\hline $\begin{array}{l}\text { CIC05192 (V.11) } \\
\text { unaffected nephew }\end{array}$ & & no & no & & \\
\hline CIC00009 (F8)* & 4 & c.2169delA & p.Ile725TyrfsX13 nd & na & na \\
\hline $\begin{array}{l}\text { CIC00010 } \\
\text { affected son }\end{array}$ & & c. $2169 \mathrm{delA}$ & p.Ile725TyrfsX13 & & \\
\hline $\begin{array}{l}\mathrm{CIC} 02805 \text { (F1039) } \\
\text { (II.4) }\end{array}$ & 4 & c.2169delA & p.Ile725TyrfsX13 nd & na & na \\
\hline $\begin{array}{l}\text { CIC01239 (F749) } \\
\text { (III.3) }\end{array}$ & 4 & c. $2275 \mathrm{~A}>\mathrm{T}$ & p.Arg759X & na & na \\
\hline $\begin{array}{l}\text { CIC04884 (II.3) } \\
\text { affected mother }\end{array}$ & & c. $2275 \mathrm{~A}>\mathrm{T}$ & p.Arg759X & & \\
\hline
\end{tabular}


*This family was also investigated by another French group (Christian Hamel, U583,

INSERM, Institute for Neurosciences of Montpellier, France) and thus further details are given elsewhere.

Supplementary Table 2: Previously described RP1 variants with unclear pathogenicity

\begin{tabular}{|c|c|c|c|c|c|}
\hline Disorder & Exon & Nucleotide Exchange & Protein Effect & $\begin{array}{l}\text { Why } \\
\text { unclear/comment }\end{array}$ & Literture \\
\hline $\operatorname{adRP}$ & 3 & c. $652 \mathrm{G}>\mathrm{A}$ & p.Ala218Thr & $\begin{array}{l}\text { No other family } \\
\text { members } \\
\text { available for co- } \\
\text { segregation }\end{array}$ & $\begin{array}{l}\text { (Berson et al. } \\
2001 \text { ) }\end{array}$ \\
\hline adRP & 4 & c. $1222 \mathrm{~A}>\mathrm{C}$ & p.Ile408Leu & $\begin{array}{l}\text { PolyPhen and } \\
\text { SIFT benign but } \\
\text { did not appear in } \\
190 \text { controls }\end{array}$ & $\begin{array}{l}\text { (Zhang et al. } \\
\text { 2010) }\end{array}$ \\
\hline $\operatorname{adRP}$ & 4 & c. $1437 \mathrm{G}>\mathrm{T}$ & p.Met479Ile & $\begin{array}{l}\text { Insufficient data } \\
\text { to conclude } \\
\text { whether } \\
\text { associated with } \\
\text { RP }\end{array}$ & $\begin{array}{l}\text { (Baum et al. } \\
\text { 2001; Chiang et } \\
\text { al. 2006) }\end{array}$ \\
\hline adRP? & 4 & c. $1989 \mathrm{G}>\mathrm{T}$ & p.Lys663Asn & $\begin{array}{l}\text { no other family } \\
\text { members for } \\
\text { testing, non- } \\
\text { conserved residue } \\
\text { in human and } \\
\text { mouse }\end{array}$ & $\begin{array}{l}\text { (Bowne et al. } \\
\text { 1999; Payne et } \\
\text { al. 2000) }\end{array}$ \\
\hline adRP & 4 & $\begin{array}{l}\text { c. } 2376 \mathrm{~A}>\mathrm{C} \\
\text { c. } 2393 \mathrm{G}>\mathrm{C}\end{array}$ & $\begin{array}{l}\text { p.Lys792Gln } \\
\text { p.Arg798Asn }\end{array}$ & $\begin{array}{l}\text { no other family } \\
\text { members, Lys } \\
\text { conserved, Arg } \\
\text { not }\end{array}$ & $\begin{array}{l}\text { (Payne et al. } \\
\text { 2000) }\end{array}$ \\
\hline adRP & 4 & c. $2700 \mathrm{~A}>\mathrm{C}$ & p.Lys900Thr & $\begin{array}{l}\text { conserved residue, } \\
\text { no other family } \\
\text { members }\end{array}$ & $\begin{array}{l}\text { (Payne et al. } \\
2000)\end{array}$ \\
\hline $\operatorname{adRP}$ & 4 & c. $2707 \mathrm{~T}>\mathrm{A}$ & p.Pro903Leu & $\begin{array}{l}2 \text { families, } 1.2 \\
\text { affected carried } \\
\text { the mutation, } \\
\text { while the } \\
\text { unaffected did not } \\
\text { show the } \\
\text { mutation. } 2.1 \\
\text { affected and one } \\
\text { at-risk individual } \\
\text { incomplete }\end{array}$ & $\begin{array}{l}\text { (Sheng et al. } \\
\text { 2008) }\end{array}$ \\
\hline
\end{tabular}




\begin{tabular}{|c|c|c|c|c|c|}
\hline & & & \multicolumn{3}{|c|}{ penetrance } \\
\hline $\mathrm{RP}$ & 4 & c. $4955 \mathrm{G}>\mathrm{T}$ & p.Arg1652Leu & $\begin{array}{l}\text { Conserved } \\
\text { residue, no other } \\
\text { family member }\end{array}$ & $\begin{array}{l}\text { (Zhang et al. } \\
\text { 2010) }\end{array}$ \\
\hline adRP & 4 & c. $5377 \mathrm{C}>\mathrm{T}$ & p.Pro1793Ser & $\begin{array}{l}\text { patient carried } \\
\text { also a nonsense } \\
\text { mutation in cis, } \\
\text { which was } \\
\text { considered to be } \\
\text { pathogenic }\end{array}$ & $\begin{array}{l}\text { (Payne et al. } \\
\text { 2000; Berson et } \\
\text { al. 2001) }\end{array}$ \\
\hline adRP? & 4 & c.5423T $>C$ & p.Leu1808Pro & $\begin{array}{l}\text { no other family } \\
\text { members for } \\
\text { testing }\end{array}$ & $\begin{array}{l}\text { (Bowne et al. } \\
1999 \text { ) }\end{array}$ \\
\hline adRP & 4 & c. $6338 \mathrm{C}>\mathrm{A}$ & p.Thr2113Asn & $\begin{array}{l}\text { no other family } \\
\text { members for } \\
\text { testing }\end{array}$ & $\begin{array}{l}\text { (Payne et al. } \\
\text { 2000; Sohocki } \\
\text { et al. 2001) }\end{array}$ \\
\hline
\end{tabular}

Supplementary Table 3: Previously described rare $R P 1$ variants which are unlikely to be pathogenic

\begin{tabular}{|c|c|c|c|c|c|}
\hline Disorder & Exon & Nucleotide Exchange & Protein Effect & Why & Literture \\
\hline adRP & 2 & c. $502 C>G$ & p.Arg168Gly & $\begin{array}{l}\text { did not co- } \\
\text { segregate }\end{array}$ & $\begin{array}{l}\text { (Berson et al. } \\
2001 \text { ) }\end{array}$ \\
\hline $\mathrm{RP}$ & 3 & c.746G $>A$ & p.Arg249His & $\begin{array}{l}\text { did not co- } \\
\text { segregate }\end{array}$ & $\begin{array}{l}\text { (Baum et al. } \\
\text { 2001; Chiang et } \\
\text { al. 2006) }\end{array}$ \\
\hline $\mathrm{RP}$ & intron 3 & c. $787+34 \mathrm{~T}>\mathrm{C}$ & - & $\begin{array}{l}\text { no evidence } \\
\text { to be } \\
\text { pathogenic }\end{array}$ & $\begin{array}{l}\text { (Zhang et al. } \\
\text { 2010) }\end{array}$ \\
\hline $\mathrm{RP}$ & 4 & c. $2116 \mathrm{G}>\mathrm{C}$ & p.Gly706Arg & $\begin{array}{l}\text { appeared in } \\
\text { controls }\end{array}$ & $\begin{array}{l}\text { (Baum et al. } \\
\text { 2001; Chiang et } \\
\text { al. 2006; Zhang } \\
\text { et al. 2010) }\end{array}$ \\
\hline adRP & 4 & c. $2255 \mathrm{C}>\mathrm{T}$ & p.Thr752Met & $\begin{array}{l}\text { did not co- } \\
\text { segregate, } \\
\text { appeared in } \\
\text { controls }\end{array}$ & $\begin{array}{l}\text { (Roberts et al. } \\
2006)\end{array}$ \\
\hline adRP & 4 & c. $2953 \mathrm{~A}>\mathrm{T}$ & p.Asn985Tyr & $\begin{array}{l}\text { Segregated in } \\
\text { one family } \\
\text { but also } \\
\text { reported as a }\end{array}$ & $\begin{array}{l}\text { (Sullivan et al. } \\
\text { 1999; Sohocki et } \\
\text { al. 2001; Sheng } \\
\text { et al. 2008) }\end{array}$ \\
\hline
\end{tabular}




\begin{tabular}{|c|c|c|c|c|c|}
\hline & & & & $\begin{array}{l}\text { SNP } \\
\text { rs2293869 } \\
\text { and in } \\
\text { specific } \\
\text { cohorts } \\
\text { appeared in } \\
\text { controls } \\
\end{array}$ & \\
\hline$\overline{\mathrm{adRP}}$ & 4 & c. $3215 A>G$ & p.Asp1072Gly & $\begin{array}{l}\text { did not co- } \\
\text { segregate }\end{array}$ & $\begin{array}{l}\text { (Berson et al. } \\
2001)\end{array}$ \\
\hline $\mathrm{RP}$ & 4 & c. $3024 \mathrm{G}>\mathrm{A}$ & p.Gln1008Gln & $\begin{array}{l}\text { appeared in } \\
\text { controls }\end{array}$ & $\begin{array}{l}\text { (Baum et al. } \\
\text { 2001; Zhang et } \\
\text { al. 2010) }\end{array}$ \\
\hline $\mathrm{RP}$ & 4 & $\begin{array}{l}\text { Wrong annotation, not clear } \\
\text { previously: c. } 3188 \mathrm{G}>\mathrm{A}\end{array}$ & p.Gln1063Arg & $\begin{array}{l}\text { appeared in } \\
\text { controls }\end{array}$ & $\begin{array}{l}\text { (Baum et al. } \\
\text { 2001; Chiang et } \\
\text { al. 2006) }\end{array}$ \\
\hline adRP & 4 & c. $4067 \mathrm{~T}>\mathrm{C}$ & p.Leu1356Ser & $\begin{array}{l}\text { did not co- } \\
\text { segregate }\end{array}$ & $\begin{array}{l}\text { (Berson et al. } \\
\text { 2001) }\end{array}$ \\
\hline $\operatorname{adRP}$ & 4 & c. $4250 \mathrm{~T}>\mathrm{C}$ & p.Leu1417Pro & $\begin{array}{l}\text { did not co- } \\
\text { segregate, } \\
1 / 95 \text { controls }\end{array}$ & $\begin{array}{l}\text { (Berson et al. } \\
\text { 2001) }\end{array}$ \\
\hline adRP & 4 & c. $4784 \mathrm{G}>\mathrm{A}$ & p.Arg1595Gln & $\begin{array}{l}\text { no other } \\
\text { family } \\
\text { members } \\
\text { now } \\
\text { documented } \\
\text { as SNP } \\
\text { rs35084330 } \\
\end{array}$ & $\begin{array}{l}\text { (Bowne et al. } \\
\text { 1999; Payne et } \\
\text { al. 2000; } \\
\text { Sohocki et al. } \\
\text { 2001) }\end{array}$ \\
\hline controls & 4 & c. $5797 \mathrm{C}>\mathrm{T}$ & p.Arg1933X & $\begin{array}{l}\text { appeared in } \\
\text { controls }\end{array}$ & $\begin{array}{l}\text { (Baum et al. } \\
\text { 2001; Chiang et } \\
\text { al. 2006) }\end{array}$ \\
\hline$\overline{\mathrm{adRP}}$ & 4 & c. $.5805 \mathrm{~T}>\mathrm{G}$ & p.Phe1935Leu & $\begin{array}{l}\text { did not co- } \\
\text { segregate }\end{array}$ & $\begin{array}{l}\text { (Berson et al. } \\
2001)\end{array}$ \\
\hline $\mathrm{RP}$ & 4 & c. $6045 \mathrm{~A}>\mathrm{G}$ & p.Leu2015Leu & $\begin{array}{l}\text { no evidence } \\
\text { to be } \\
\text { pathogenic }\end{array}$ & $\begin{array}{l}\text { (Zhang et al. } \\
\text { 2010) }\end{array}$ \\
\hline adRP & 4 & c. $6196 \mathrm{G}>\mathrm{A}$ & p.Asp2066Asn & $\begin{array}{l}\text { did not co- } \\
\text { segregate, } \\
1 / 91 \text { controls }\end{array}$ & $\begin{array}{l}\text { (Berson et al. } \\
\text { 2001) }\end{array}$ \\
\hline controls & 4 & c. $6423 \mathrm{~A}>\mathrm{G}$ & p.Ile2141Met & $\begin{array}{l}\text { appeared in } \\
\text { controls }\end{array}$ & $\begin{array}{l}\text { (Baum et al. } \\
\text { 2001; Chiang et } \\
\text { al. 2006) }\end{array}$ \\
\hline $\mathrm{RP}$ & 4 & c. $6542 C>T$ & - & $\begin{array}{l}\text { appeared in } \\
\text { controls }\end{array}$ & $\begin{array}{l}\text { (Baum et al. } \\
\text { 2001; Zhang et }\end{array}$ \\
\hline
\end{tabular}


Supplementary Table 4: Rare $R P 1$ variants with uncertain pathogenicity in a French adRP cohort.

\begin{tabular}{|c|c|c|c|c|c|c|c|}
\hline \multicolumn{2}{|c|}{ Index (families) } & Exon & Nucleotide Exchange & Protein Effect & controls & SIFT & POLYPHEN \\
\hline \multicolumn{2}{|c|}{$\begin{array}{l}\text { CIC01234 (F745) } \\
\text { (III.4) } \\
\text { CIC04861 (IV.8) } \\
\text { unaffected niece }\end{array}$} & 4 & $\begin{array}{l}\text { c. } 4193 \mathrm{C}>\mathrm{G} \\
\text { no }\end{array}$ & $\begin{array}{l}\text { p.Ser1398Cys } \\
\text { no }\end{array}$ & 0/190 & $\begin{array}{l}\text { affect } \\
\text { protein } \\
\text { function }\end{array}$ & $\begin{array}{l}\text { possibly } \\
\text { damaging }\end{array}$ \\
\hline \multicolumn{2}{|c|}{$\begin{array}{l}\text { CIC04983, (III.2) } \\
\text { assymptomatic } \\
\text { brother }\end{array}$} & & c. $4193 \mathrm{C}>\mathrm{G}$ & p.Ser1398Cys & & & \\
\hline \multicolumn{8}{|c|}{ Supplementary Table 5: Rare $R P 1$ variants in a French adRP cohort. } \\
\hline $\begin{array}{l}\text { Index } \\
\text { (families) }\end{array}$ & Exon & & otide Exchange & Protein Effect & controls & SIFT & POLYPHEN \\
\hline $\begin{array}{l}\text { CIC00869 } \\
\text { (F558) }\end{array}$ & 1 & & $\mathrm{~A}>\mathrm{G}$ & - & nd & na & na \\
\hline $\begin{array}{l}\text { Other } \\
\text { CIC04860 } \\
\text { affected } \\
\text { daughter }\end{array}$ & 1 & no & & & & & \\
\hline $\begin{array}{l}\text { CIC02802 } \\
\text { (F933) }\end{array}$ & 4 & & $9 \mathrm{G}>\mathrm{A}$ & p.Val12047Met & nd & tolerated & benign \\
\hline $\begin{array}{l}\text { CIC03164 } \\
\text { affected son }\end{array}$ & 4 & no & & no & & & \\
\hline $\begin{array}{l}\text { CIC03224 } \\
\text { unaffected } \\
\text { daughter }\end{array}$ & 4 & no & & no & & & \\
\hline $\begin{array}{l}\text { CIC03420 } \\
\text { unaffected } \\
\text { son }\end{array}$ & 4 & no & & no & & & \\
\hline CIC02630 & 4 & no & & no & & & \\
\hline
\end{tabular}


affected

cousin

\begin{tabular}{llllll}
\hline CIC01239 & 4 & c.5962A $>$ G & p.Ile1988Val nd & tolerated benign \\
$($ F749)
\end{tabular}

Nucleotide numbering reflects cDNA numbering with +1 corresponding to the A of the ATG translation initiation codon in the reference sequence NM_006269.1, according to journal guidelines (www.hgvs.org/mutnomen). The initiation codon is codon 1. 